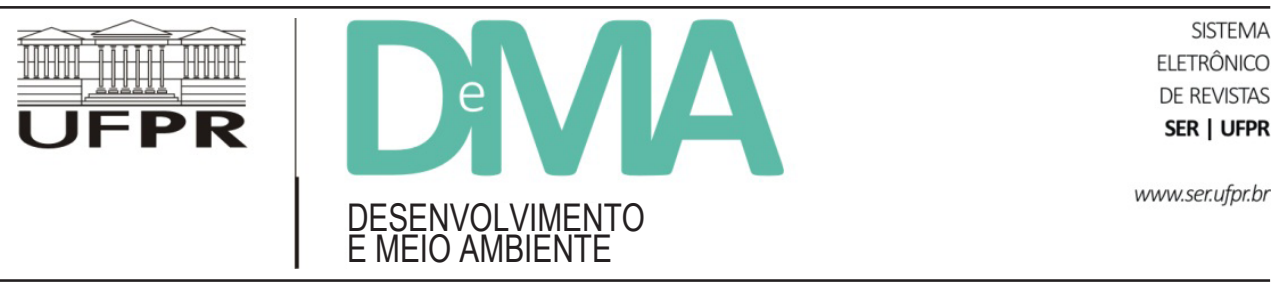

\title{
Uso da bicicleta como meio de transporte nos estudos pessoa-ambiente
}

\section{Bicycle as a Mean of Transportation on Person-Environment Studies}

\author{
Nikolas OLEKSZECHEN ${ }^{1 *}$, Marcia BATTISTON ${ }^{1}$, Ariane KUHNEN ${ }^{1}$ \\ ${ }^{1}$ Programa de Pós-Graduação em Psicologia, Universidade Federal de Santa Catarina (UFSC), Florianópolis, SC, Brasil. \\ *E-mail de contato: nikolas.oleks@gmail.com
}

Artigo recebido em 24 de outubro de 2015, versão final aceita em 11 de abril de 2016.

RESUMO: Diante do desafio da mobilidade urbana nas grandes cidades, formuladores de políticas públicas e pesquisadores têm sugerido a bicicleta como alternativa à questão. Assim, este artigo busca verificar como os estudos pessoa-ambiente enfocam a temática do uso de bicicletas como meio de transporte. Realizou-se revisão sistemática da literatura com estudos nacionais e internacionais publicados entre 2009 e 2014, buscando identificar o enfoque dado ao uso de bicicleta, a natureza dos estudos e os instrumentos utilizados. 33 artigos corresponderam às buscas e foram subdivididos em três categorias: hábitos e atitudes, ambiente e comportamento, e percepção. Aponta-se para a primazia de estudos internacionais de natureza quantitativa, com uso de questionários e escalas. Os resultados se aproximam ao atrelarem o uso da bicicleta ao bem-estar e divergem ao relacionarem o nível socioeducacional à escolha por esse modal. Aponta-se para a necessidade de compreender o fenômeno de maneira multideterminada, levando em consideração aspectos individuais, perceptivos, socioculturais e ambientais referentes ao tema.

Palavras-chave: psicologia ambiental; ambientes urbanos; transportes; revisão de literatura; bicicleta.

ABSTRACT: In face of the urban mobility challenge in big cities, public policy makers and researchers suggest the bicycle commuting as an alternative to deal with this issue. This article aims to verify how the person-environment studies treat the use of bicycles as a means of transportation topic. A systematic review of literature was done with national and international studies published between 2009 and 2014, aiming to identify the methodological approach for bicycle commuting in person-environment studies, the nature of the researches and the instruments utilized. Thirty three articles satisfied the research and were subdivided into three categories: habits and attitudes, environment and behavior, and perception. The primacy of international studies with quantitative approach, using questionnaires and scales is noticeable. The results approximate when articulate bicycle commuting to well-being and diverge when relate socio-educational level to modal choice. These results indicate the necessity to comprehend the phenomenon in a multidetermined way, taking into consideration individual, perceptive, sociocultural, and environmental aspects concerning the topic.

Keywords: environmental psychology; urban environments; transportation; literature review; bicycle. 


\section{Introdução}

Diante do desafio de se locomover nas cidades e promover o deslocamento de pessoas de maneira sustentável, o conceito de mobilidade urbana aparece com frequência nas agendas políticas. No contexto brasileiro, um marco legal recente a esse respeito é a lei que institui as diretrizes da Política Nacional de Mobilidade Urbana (Brasil, 2012). Com base nela, entende-se mobilidade urbana como a condição em que se realizam deslocamentos de pessoas ou de cargas em espaços urbanos. Sua implementação objetiva contribuir para o acesso universal às cidades, bem como promover o desenvolvimento sustentável pela priorização dos modos de transporte não motorizados sobre os motorizados (Brasil, 2012).

Nesse sentido, o uso da bicicleta como meio de transporte tem demonstrado ser benéfico coletiva e individualmente, uma vez que oferece vantagens à saúde de quem pedala pela prática de exercício físico e por ser um modo barato e rápido de se locomover quando comparado a outros meios. Além disso, o baixo impacto ambiental causado e o baixo custo de infraestrutura são benefícios atrelados ao seu uso, o que tem chamado a atenção dos formuladores de políticas públicas e pesquisadores. Heinen et al. (2010) destacam que a quantidade de pesquisas acadêmicas a respeito do uso de bicicleta tem crescido, ainda que sua visibilidade seja baixa quando comparada a outros meios de transporte.

\subsection{Mobilidade nos estudos pessoa-ambiente}

No que tange aos estudos pessoa-ambiente, campo de investigação interdisciplinar que busca compreender as transações das pessoas com seus entornos, destaca-se a Psicologia Ambiental como a disciplina atenta principalmente à relação de reciprocidade entre esses elementos, sendo este o foco de seu interesse (Günther, 2003).

Para além do reflexo de orientação, a mobilidade é destacada por Günther (2003) como essencial para o desenvolvimento e o bem-estar humanos. O grau de mobilidade afeta tanto o acesso a materiais e ideias importantes, subjetiva e objetivamente, quanto as possibilidades de ação que o ambiente propicia para aquele que com ele está em interação. Portanto, a mobilidade, ou a circulação pelos espaços físicos que favorecem a exploração e a interação, bem como as possibilidades oferecidas pelos ambientes para essa interação, são destacadas como cerne dos estudos pessoa-ambiente (Günther, 2003).

Ao pensar a mobilidade em um contexto específico como o das cidades, faz-se necessário que os diferentes modais de transporte possam ser otimizados visando à fluidez na circulação e o acesso para as diferentes possibilidades que o ambiente propicia. Seja por meio do transporte motorizado (carros, motocicletas, ônibus, entre outros), seja pelo transporte ativo (de bicicleta ou a pé), a mobilidade nas cidades vem sendo estudada levando-se em conta também as questões de sustentabilidade e a escolha dos modais, resultando no que se denomina de mobilidade urbana sustentável (Vasconcellos, 2012; Villada \& Portugal, 2015). Esse conceito enfatiza os atributos qualitativos dos deslocamentos, com destaque aos modais que provocam menor impacto social e ambiental (Amorim et al., 2014; Villada \& Portugal, 2015).

A fim de identificar as principais produções acadêmicas no campo da mobilidade urbana por meio do uso de bicicletas como alternativa de transporte nos estudos pessoa-ambiente, foi realizada uma revisão sistemática da literatura sobre o tema. Buscou-se responder aos seguintes questio- 
namentos: a) Como o uso de bicicleta vem sendo enfocado nos estudos pessoa-ambiente?; b) Qual a natureza dessas pesquisas e quais os principais instrumentos utilizados?

\section{Método}

Recorreu-se às bases de dados Science Direct, Sage, PePSIC e SciELO por congregarem periódicos internacionais e nacionais. Science Direct e Sage indexam os principais periódicos da psicologia ambiental (Journal of Environmental Psychology e Environment and Behavior, respectivamente), e as bases PePSIC e SciELO, os principais periódicos brasileiros. O parâmetro cronológico utilizado foi o período compreendido entre os anos de 2009 e 2014, por visar às produções referentes aos últimos cinco anos. Foi realizada no período entre 16 e 19/09/2014 com os descritores psicologia ambiental (environmental psychology), bicicleta (bicycle) bike) e transporte (commuting ${ }^{1}$ ), articulados com os booleanos AND e OR.

Assim, nas bases de dados estrangeiras a busca foi feita com: environmental psychology AND (bicycl* OR bik*), environmental psychology AND [commut* AND (bicycl* OR bik*)]. Nas bases de periódicos nacionais recorreu-se às combinações: psicologia ambiental AND bicicleta, psicologia AND bicicleta e transporte AND bicicleta. Ao realizar buscas em bases de periódicos nacionais com descritores análogos ao idioma inglês, não foram obtidos resultados. Desse modo, optou-se pela inclusão do descritor psicologia a fim de aumentar a abrangência das buscas.
Inicialmente, 429 artigos corresponderam às buscas e foram submetidos a critérios de inclusão e exclusão. Como o foco do estudo foram artigos que tratassem da relação entre comportamento do ciclista e ambiente, foram incluídos os artigos que: a) tivessem disponível seu texto completo ${ }^{2} ;$ b) disponíveis nos idiomas inglês ou português; e c) descrevessem estudos empíricos a respeito da relação pessoa-ambiente ou comportamentos de ciclistas. Foram excluídos da busca artigos que: a) fossem teóricos ou de revisão; b) tratassem exclusivamente da comparação e da transferência entre meios de transportes (como transporte público, carro, pedestres, etc.), dos impactos políticos e econômicos do uso da bicicleta como meio de transporte, de acidentes e impactos do ciclismo para a saúde física, e de sistemas de compartilhamento de bicicletas.

Após a aplicação dos critérios, 33 artigos corresponderam à pesquisa e passaram por leitura integral do texto. $\mathrm{O}$ foco da leitura recaiu sobre a relação pessoa-ambiente e o comportamento do ciclista, bem como os métodos e instrumentos utilizados na abordagem do uso da bicicleta como meio de transporte. Os artigos foram organizados e agrupados em três categorias, de acordo com a temática abordada e os objetivos, sendo elas: hábitos e atitudes; ambiente e comportamento; e percepção, conforme Tabela 1.

Atitudes e comportamentos compõem categorias distintas por se tratarem de fenômenos diferentes. Entende-se como atitude uma maneira organizada de pensar e sentir sobre diversos temas ou questões, sobre outras pessoas ou acontecimentos. Indica um estado interior de disposição

\footnotetext{
1 O termo commuting, em inglês, caracteriza o ato de se deslocar de casa ao trabalho ou ao local de ensino. Seu correspondente em português se aproxima de "movimento pendular" ou "transporte pendular". Neste trabalho será utilizada a palavra transporte.

2 Optou-se pela inclusão dos textos completos pela necessidade de acesso aos detalhes teórico-metodológicos dos estudos. Em relação à escolha das bases de dados, reforça-se o fato de os principais periódicos da psicologia ambiental estarem indexados nestas, ainda que o acesso aos textos esteja atrelado à assinatura por parte da Instituição de Ensino Superior de filiação.
} 
à ação, mas não a ação em si. São definidas como uma organização de crenças e cognições dotadas de carga afetiva favorável ou não a um objeto social definido, que predispõe a uma ação compatível com essas cognições e afetos (Rodrigues et al., 1999). O comportamento, por sua vez, se refere ao ato em si e não somente a um ponto de vista pessoal. Trata-se de uma forma de agir no cotidiano, um conjunto de procedimentos ou reações que resultam das interações entre as pessoas e o seu ambiente (Doron \& Parot, 2001).

TABELA 1 - Divisão dos artigos de acordo com categorias de análise.

\begin{tabular}{|c|c|}
\hline Categoria & Referências \\
\hline $\begin{array}{l}\text { Hábitos e } \\
\text { atitudes }\end{array}$ & $\begin{array}{l}\text { Araújo et al. (2009a; 2009b); Daley \& } \\
\text { Rissel (2011); de Bruijn et al. (2009); } \\
\text { Eryiğit \& Ter (2014); Forward (2014); } \\
\text { Franco et al. (2014); Gatersleben \& } \\
\text { Haddad (2010); Hansen \& Nielsen } \\
\text { (2014); Heinen et al. (2011); Kienteka } \\
\text { et al. (2014); Nkurunziza et al. (2012); } \\
\text { Passafaro et al. (2014); Sherwin et al. } \\
\text { (2014); Underwood et al. (2014); Woo } \\
\text { et al. (2010); Wooliscroft \& Ganglmair- } \\
\text { Wooliscroft (2014) }\end{array}$ \\
\hline $\begin{array}{c}\text { Ambiente e } \\
\text { comportamento }\end{array}$ & $\begin{array}{l}\text { Basu \& Vasudevan (2013); Buehler (2012); } \\
\text { Flynn et al. (2012); Sallis et al. (2013); } \\
\text { Spencer et al. (2013); Vandenbulcke et al. } \\
\text { (2011); Willis et al. (2013); Zhang et al. } \\
\text { (2014); Zhao (2014) }\end{array}$ \\
\hline Percepção & $\begin{array}{l}\text { Chataway et al. (2014); de Waard et al. } \\
\text { (2011; 2014); Kakefuda et al. (2009); } \\
\text { Kienteka et al. (2012); McIlveny (2014); } \\
\text { Vansteenkiste } \text { et al. (2014) }\end{array}$ \\
\hline
\end{tabular}

FONTE: Busca em bases de dados (2014).

\section{Resultados}

\subsection{Hábitos e atitudes relacionados ao uso da bicicleta}

Esta categoria reúne 17 estudos a respeito das influências sociais do uso da bicicleta e compreende desde aspectos da esfera individual, como a força dos hábitos e das emoções na adesão à bicicleta como meio de transporte, até as influências de valores culturais em seu uso. De maneira geral, entende-se que a execução repetida de um comportamento constitui um hábito, enquanto atitude representa a postura geral da pessoa de ser favorável ou não a dado objeto (Ajzen, 1991).

A respeito dos hábitos, de Bruijn, Kremers et al. (2009) exploram como sua força se relaciona ao uso da bicicleta como meio de transporte em adultos de uma cidade holandesa. A partir da aplicação de questionário e escalas, identificaram que a força do hábito está positivamente relacionada ao uso de bicicleta, o que indica a possibilidade de esse comportamento se tornar habitual. Por outro lado, quando o hábito de uso da bicicleta ainda não está instaurado ou é fraco, a intenção de usá-la é forte, indicando que o hábito está intimamente relacionado à intenção, ou seja, pessoas que não utilizam a bicicleta têm uma forte inclinação para começar a fazê-lo.

A influência dos hábitos também é considerada, ao lado de atitudes, emoções e normas sociais, como preditor do desejo de pedalar (Passafaro et al., 2014). Em estudo conduzido em uma grande cidade italiana, objetivou-se identificar as implicações cognitivas e afetivas na escolha do uso da bicicleta. Com uso de questionário autoadministrado e análises estatísticas dos dados, Passafaro et al. (2014) apontam que os principais preditores do desejo de usar bicicleta são emoções antecipadas e o comportamento passado, ao passo que normas sociais e atitudes predizem indiretamente. Dessa maneira, sentir-se feliz, relaxado e satisfeito com a possibilidade de usar a bicicleta e tê-la usado anteriormente (indicação de hábito), por exemplo, são fatores que podem indicar seu uso futuro. 
Atrelado ao hábito, um estudo buscou analisar os impactos dos valores culturais no uso da bicicleta em uma cidade turca (Eryiğit \& Ter, 2014). Neste, o hábito da pedalada se associa com variáveis demográficas como idade, posse da bicicleta (não ter bicicleta mostra-se como motivo para não pedalar) e com pressões sociais. Assim, Eryiğit \& Ter (2014) ressaltam que fatores sociais relacionados à imagem da bicicleta em uma cultura são relevantes no desenvolvimento do hábito, ao indicarem que pessoas podem não aderir ao uso da bicicleta, por exemplo, se seu contexto social defende que esse é um hábito "estranho", pouco familiar, que não se encaixa naquele contexto ou cultura.

Os fatores sociais foram foco de análise em estudo qualitativo conduzido a fim de compreender como as influências sociais afetam na decisão de começar a pedalar (Sherwin et al., 2014). Por meio de entrevistas individuais semiestruturadas com ciclistas ingleses, Sherwin et al. (2014) corroboram os resultados de Eryiğit \& Ter (2014) ao concluírem que o entorno social, juntamente com família e amigos, é um fator importante na decisão de usar a bicicleta.

No mesmo sentido, Daley \& Rissel (2011) exploram as perspectivas e imagens do uso da bicicleta em uma cidade australiana. Com enfoque qualitativo, as autoras realizaram grupos focais com ciclistas e não ciclistas e identificaram que fatores como sustentabilidade (conduta "limpa", "verde"), saúde e riscos de acidentes estão ligados às imagens do uso da bicicleta. Em relação ao ciclista, as imagens relacionadas são aquelas de pessoas que se arriscam, que burlam regras e que se enquadram em subculturas, ou seja, em modos não hegemônicos de circular na cidade como os motorizados. Com isso, valores culturais, influências e imagens sociais a respeito do uso de bicicletas se evidenciam como fatores que afetam diretamente o uso (ou não) da bicicleta.
A respeito da imagem social da bicicleta, Gatersleben \& Haddad (2010) investigaram os pontos de vista em relação ao "ciclista típico" em cidades inglesas e verificaram se esse olhar se relaciona com o comportamento ou intenção de pedalar. A aplicação de questionários e escalas, aliada à análise estatística, demonstrou que se tende a perceber o ciclista, naquele contexto, em quatro estereótipos: ciclistas responsáveis, que utilizam a bicicleta de maneira responsável e segura; ciclista como estilo de vida, que contempla ciclistas que gastam tempo e dinheiro com bicicleta; commuters, pessoas que usam a bicicleta para ir ao trabalho independente das condições climáticas; hippy-go-lucky (despreocupada, alegre), geralmente pessoas gentis que usam a bicicleta no dia a dia, além de ir ao trabalho. As autoras apontam para a existência de diferenças na maneira como ciclistas e não ciclistas enxergam o uso da bicicleta e atentam para as implicações sociais nessa prática.

Em relação aos fatores que motivam os indivíduos a pedalarem, os estudos identificaram que características individuais, ambientais e políticas exercem forte influência de modo a facilitar ou dificultar o uso da bicicleta. Nkurunziza et al. (2012) identificaram que o status social do ciclista, insegurança e desconforto ao pedalar interferem negativamente no uso da bicicleta em uma cidade da Tanzânia. Por outro lado, ao se comparar ciclistas que pedalam curtas e longas distâncias até o trabalho, aqueles que percorrem longas distâncias se motivam a fazê-lo por razões pessoais e indicam as experiências positivas, humor e diminuição do estresse como benefícios da pedalada (Hansen \& Nielsen, 2014).

Heinen et al. (2011) ressaltam que as atitudes estão envolvidas na decisão de usar a bicicleta em uma cidade holandesa. Os indivíduos amparam sua escolha com base nos benefícios proporcionados, 
como tempo, conforto e flexibilidade. Além disso, preocupações com o meio ambiente (Heinen et al., 2011; Forward, 2014), fatores políticos como redução do custo da bicicleta e de taxas de importação (Nkurunziza et al., 2012) e preço do combustível (Wooliscroft \& Ganglmair-Wooliscroft, 2014), bem como características demográficas de renda e moradia (Franco et al., 2014) e idade (Eryiğit \& Ter, 2014; Kienteka et al., 2014) são aspectos importantes na opção pela bicicleta.

Dentre as características demográficas, aquela que apareceu com maior frequência foi sexo, associado à possibilidade de mulheres perceberem maiores barreiras para pedalar (Kienteka et al., 2014), a fatores como gostar de pedalar e a prática de exercício (Araújo et al., 2009a) - em estudos conduzidos em duas cidades brasileiras diferentes - e às imagens negativas e estigmas do uso da bicicleta em uma cidade estadunidense (Underwood et al., 2014).

Além disso, quando relacionado com o papel das emoções na escolha da bicicleta, Woo et al. (2010) identificam que em uma cidade da Nova Zelândia homens pedalam mais do que mulheres, ainda que estas relatem maior suporte de políticas públicas, desvantagem no uso do carro e compromisso pró-ambiental. Para os autores, isso possivelmente se dá porque aqueles que pedalam visam aos prazeres sensoriais e somáticos da atividade (principalmente no caso dos homens) em detrimento dos fatores cognitivos, como benefício pessoal, social ou ecológico, no caso das mulheres.

De maneira geral, os trabalhos referentes à categoria dos hábitos e atitudes contribuem na compreensão dos fatores individuais e sociais ao se optar pela bicicleta como meio de transporte. Considera-se a importância de fatores como rapidez e eficiência (Araújo et al., 2009b; Hansen \&
Nielsen, 2014), gostar de andar de bicicleta (Araújo et al., 2009b), uso da bicicleta como atividade de lazer (Kienteka et al., 2014), emoções (Woo et al., 2010; Passafaro et al., 2014) e imagem social da bicicleta e do ciclista (Gatersleben \& Haddad, 2010; Daley \& Rissel, 2011; Underwood et al., 2014), o que aponta para a multideterminação do fenômeno.

No entanto, alguns estudos apresentaram resultados inconclusivos ou contrários a outros. Em relação à qualidade de vida, por exemplo, Hansen \& Nielsen (2014) relatam, em seu estudo conduzido na Dinamarca, que um dos ganhos de se pedalar é o benefício psicológico, como percepção de experiências positivas, diminuição do estresse e melhora no humor. Por outro lado, Kineteka et al. (2014) encontraram associação inversa entre qualidade de vida e uso de bicicleta em uma cidade do sul do Brasil. Estes autores apoiam-se na possibilidade de, nesse estudo, a qualidade de vida retratar as condições gerais de vida e não exclusivamente relacionada à sua utilização.

Além disso, ao considerar o conforto ao pedalar como variável de análise, Nkurunziza et al. (2012) citam o desconforto como barreira, enquanto Heinen et al. (2011) apontam o conforto como fator que influencia positivamente. Assim, ao se compreender o uso da bicicleta de maneira contextualizada, atravessado por características que extrapolam a esfera pessoal, deve-se lançar o olhar às particularidades dos locais onde as pesquisas são conduzidas e levar em consideração, além das práticas ciclísticas particulares de cada cultura, a infraestrutura existente e as condições de materiais para a circulação. Em suma, é necessário considerar as influências do ambiente (com suas peculiaridades geográficas, sociais, culturais, etc.) na escolha da bicicleta como meio de transporte. 


\subsection{Ambiente e comportamento dos ciclistas}

Esta categoria reúne nove artigos que incluem nos objetivos e métodos a investigação do ambiente físico natural e/ou construído relacionado ao uso da bicicleta. Os aspectos investigados relacionam características como clima, relevo, qualidade das infraestruturas ciclísticas e o impacto dos usos feitos do solo no transporte com bicicletas.

Vandenbulcke et al. (2011) examinam fatores que influenciam no uso da bicicleta para deslocamentos diários e indicam que a presença de terrenos planos, qualidade das vias para circulação de bicicletas e o baixo risco de acidentes se associam à escolha da bicicleta em cidades belgas. No contexto indiano, faixas exclusivas para ciclistas são apontadas como infraestrutura favorita daquela população e devem receber maior atenção por parte das políticas locais (Basu \& Vasudevam, 2013).

$\mathrm{O}$ uso do solo, entendido pela maneira com que as atividades e serviços se distribuem no espaço urbano, também é levado em consideração na escolha da bicicleta. Na cidade de Pequim, por exemplo, identificou-se que a baixa diversidade do uso do solo e a baixa acessibilidade se associam à redução do uso da bicicleta (Zhao, 2014). No mesmo sentido, ciclistas belgas tendem a usar mais a bicicleta quando o uso do solo é diversificado (Vandenbulcke et al. 2011). Por outro lado, quando se trata da densidade de pessoas os resultados não são congruentes, uma vez que na cidade de Pequim ela não interfere significativamente no uso de bicicleta (Zhao, 2014), enquanto que em Montreal é um fator que afeta negativamente a satisfação do ciclista (Willis et al., 2013).

Além disso, a presença de estruturas como estacionamentos seguros e chuveiros no ambiente de trabalho está relacionada ao uso de bicicletas
(Buehler, 2012; Nkurunziza et al., 2012; Wooliscroft \& Ganglmair-Wooliscroft, 2014). Franco et al. (2014) identificam que a falta de estacionamentos dificulta o uso de bicicleta, principalmente entre estudantes de uma cidade brasileira. Seu uso também é reduzido quando existe estacionamento grátis para carros no local de trabalho em uma cidade dos Estados Unidos (Buehler, 2012).

Atrelado ao ambiente construído, segurança é outra característica capaz de incentivar ou desestimular o uso da bicicleta. Zhao (2014) indica que a falta de segurança no tráfego reduz o uso da bicicleta, ao encontro dos resultados de Sallis et al. (2013), que identificam maior propensão à pedalada em uma cidade estadunidense se os ciclistas estivessem seguros em relação aos carros ao transitarem. De maneira análoga, as condições climáticas de um local se associam à segurança do ciclista. Em locais de clima frio, onde fatores ambientais como luminosidade, temperatura, vento e precipitação são preponderantes, ciclistas dos Estados Unidos relatam sentimento de pouca segurança ao trafegarem (Spencer et al., 2013).

Condição climática é um fator que afeta negativamente no uso da bicicleta (Flynn et al., 2012; Spencer et al., 2013; Eryiğit \& Ter, 2014; Franco et al., 2014). Por exemplo, em estudo sobre o impacto das condições do tempo na decisão de pedalar até o local de trabalho em uma cidade dos Estados Unidos, identificou-se que precipitação, temperatura, vento e neve são os principais impedimentos (Flynn et al., 2012). Assim, sentir calor (Franco et al., 2014) ou frio (Spencer et al., 2013) é indicado como barreira para o uso de bicicleta.

Aliada às condições climáticas, há evidências de que a estação do ano em que se pedala esteja atrelada à satisfação dos ciclistas de uma cidade canadense (Willis et al., 2013). Assim, aqueles que pedalam o ano todo são mais satisfeitos do 
que aqueles que pedalam apenas nos meses mais quentes do ano. Buehler (2012) complementa ao identificar na pesquisa realizada em uma cidade dos Estados Unidos que a bicicleta é mais utilizada durante os meses de verão.

Ao considerarem variáveis sociodemográficas, Zhang et al. (2014) analisam suas relações com a disposição de utilizar a bicicleta em uma cidade brasileira. Concluem que pessoas de classe média, com níveis educacionais elevados, que levam muito tempo caminhando até o local de trabalho e que se locomovem pouco com outros meios, são mais favoráveis à bicicleta, ao passo que motoristas de carro dificilmente aderem ao seu uso.

Nota-se, portanto, que variáveis ambientais influenciam na escolha da bicicleta como meio de transporte. Fatores relacionados à inclinação do terreno (Vandenbulcke et al., 2011; Willis et al., 2013), à presença de faixas exclusivas para ciclistas (Basu \& Vasudevan, 2013) e à qualidade das vias (Vandenbulcke et al., 2011) são importantes em seu uso. Além disso, as influências climáticas e as estações do ano aparecem nos estudos como aspecto que facilita ou dificulta na pedalada (Buehler, 2012; Spencer et al., 2013; Willis et al., 2013; Eryiğit \& Ter, 2014; Flynn et al., 2012; Franco et al., 2014).

Esses aspectos dão suporte à compreensão do uso da bicicleta enquanto fenômeno humano-ambiental, uma vez que variáveis do ambiente natural e construído se aliam às comportamentais e culturais para delinear o fenômeno. Nesse sentido, ressalta-se o estudo de Willis et al. (2013), que explora a satisfação dos ciclistas de uma cidade canadense em relação às características ambientais. Identifica-se que, ao se comparar ciclistas com usuários de outros modos de transporte, aqueles são mais satisfeitos com seu deslocamento do que estes. Além disso, independência, prazer, economia, rapidez nos deslocamentos e produção da identidade de ciclista indicam a satisfação ao utilizar a bicicleta como meio de locomoção. Portanto, aponta-se para a importância de investigações da relação ciclista-ambiente de modo multidimensional, uma vez que a aproximação da experiência de pedalar na cidade pode fornecer subsídios relevantes na compreensão das necessidades concretas dos ciclistas e subsidiar a elaboração de políticas públicas voltadas a essa população.

\subsection{Percepção do ciclista no uso da bicicleta}

Os estudos desta categoria analisam os componentes perceptivos do comportamento de pedalar. São sete estudos que investigam características referentes à percepção durante o uso da bicicleta, principalmente as visuais e auditivas. Além disso, um estudo de caráter qualitativo a respeito das relações sociais durante a pedalada foi incluído.

Dois estudos de delineamento experimental foram conduzidos em uma cidade holandesa a fim de investigar os efeitos de escutar músicas no comportamento do ciclista (de Waard et al., 2011) e os efeitos de operar um aparelho touch screen durante a pedalada (de Waard et al., 2014). No primeiro estudo, verificou-se que a velocidade da pedalada não varia sob influência do volume e da batida da música, ainda que a percepção auditiva do entorno seja negativamente afetada. Além disso, o uso do aparelho musical também interfere na diminuição da visão periférica.

No segundo estudo (de Waard et al., 2014), o uso de aparelho touch screen foi avaliado em situações de enviar mensagem, falar ao telefone e jogar. Em consonância com o estudo anterior, identificou-se que a percepção de estímulos periféricos diminui com o manuseio do aparelho, aliada à diminuição da velocidade de circulação e à variação do posiciona- 
mento na pista. Os autores chamam a atenção para o fato de que o manuseio de aparelhos eletrônicos e a influência da música durante a pedalada afetam a percepção visual e auditiva, o que limita o tempo de resposta do ciclista aos estímulos do trânsito.

A respeito da percepção de segurança do ciclista ao transitar, Chataway et al. (2014) exploram diferenças entre ciclistas de uma cidade australiana e uma dinamarquesa que pedalam em tráfego misto. Os resultados indicam que ciclistas percebem como seguras as vias separadas fisicamente do trânsito de veículos, enquanto aquelas que se situam entre o tráfego e o estacionamento de veículos são percebidas como inseguras. Os autores discutem que as percepções de segurança diferem nas duas realidades investigadas, pois estão ligadas ao contexto de cada cidade, às políticas para o uso da bicicleta nelas produzidas e da cultura decorrente disso.

A segurança do ciclista é explorada também em relação ao uso de capacete entre estudantes universitários de uma cidade dos Estados Unidos (Kakefuda et al., 2009). Identificou-se que o uso desse equipamento difere de acordo com o uso feito da bicicleta, uma vez que aqueles que pedalam por recreação percebem o capacete de maneira diferente daqueles que pedalam por transporte. De maneira geral, o uso de capacete é percebido como desconfortável, inconveniente e desnecessário para curtas distâncias. Assim, por mais que existam desvantagens em seu uso, ele é percebido como item que garante a segurança do ciclista.

Em situação experimental, Vansteenkiste et al. (2014) investigam o comportamento de ciclistas ao olharem fixamente para obstáculos de um trajeto. Nesse estudo, ciclistas belgas foram submetidos ao trânsito em ciclovias de alta e baixa qualidade e o comportamento de olhar foi captado com equipamento específico. Identificou-se que não existem diferenças na velocidade ao se pedalar nas condições experimentais, ainda que os ciclistas, nas vias de baixa qualidade, tenham mudado o foco da atenção das regiões mais distantes do ambiente para propriedades mais próximas. Conclui-se que a baixa qualidade das vias pode alterar o estado de alerta e a velocidade da resposta do ciclista em relação ao trânsito, colocando-o em perigo.

No contexto brasileiro, Kienteka et al. (2012) analisam a validade e fidedignidade de um instrumento para avaliar a percepção de barreiras no uso da bicicleta em adultos de uma cidade brasileira. Ao compararem ciclistas que pedalam por lazer e por transporte, os autores se colocam ao lado de outros estudos quantitativos que verificam barreiras no uso da bicicleta (Nkurunziza et al., 2012; Wooliscroft \& Ganglmair-Wooliscroft, 2014). Com esse instrumento, Kienteka et al. (2012) identificam que as principais barreiras para o uso da bicicleta são fatores individuais, sociais e ambientais, o que reforça a compreensão socioecológica do fenômeno.

Por fim, McIlveny (2014) conduziu estudo de caráter qualitativo na Dinamarca, com o objetivo de documentar como os ciclistas se orientam espacialmente e como as pessoas se posicionam nas vias quando em movimento. A esse fenômeno o autor deu o nome de mobile formations (formações móveis, em tradução livre para o português), que se referem também aos agrupamentos que os ciclistas fazem para se relacionarem no espaço, seja formando uma linha, um círculo ou aglomerados diversos. Interessa, portanto, o modo como os ciclistas cooperam e mantêm relações espaciais. As análises dos dados obtidos por meio de gravações de áudio e vídeo enfatizam a importância do "pedalar com", ou seja, do uso da bicicleta como uma prática social e não simplesmente como uma tarefa rotineira que inclui uma pessoa ao lado.

Os artigos incluídos nesta categoria se caracterizam pela heterogeneidade metodológica, uma 
vez que congregam três estudos de delineamento experimental e outro na perspectiva qualitativa, utilizando-se de procedimentos característicos à etnografia. Apesar disso, os estudos convergem ao enfatizarem o impacto do processo perceptivo ao pedalar, seja pelos sentidos corporais como visão e audição (de Waard et al., 2011; 2014) e suas implicações na atenção (Vansteenkiste et al., 2014), seja pela percepção do outro nas relações sociais (McIlveny, 2014). Além disso, ressalta-se a existência de uma escala validada (Kienteka et al., 2012), que pode aperfeiçoar a exploração do fenômeno do uso da bicicleta no contexto brasileiro. Esses estudos subsidiam principalmente a compreensão de fatores referentes à segurança do ciclista e às barreiras por eles encontradas no uso desse modo de transporte.

\section{Discussão e conclusão}

Identificou-se na revisão das publicações sobre uso de bicicleta como meio de transporte nos estudos pessoa-ambiente que, no período entre 2009 e 2014, os artigos se concentraram em três eixos principais dentro dos critérios adotados. Em primeiro lugar, a categoria hábitos e atitudes concentra a maior parte dos estudos e tratou das influências individuais, sociais e culturais na escolha da bicicleta. Em segundo lugar, ambiente e comportamento reúne artigos que trataram dos impactos do ambiente natural e construído no comportamento do ciclista. Por fim, estudos sobre percepção indicam os desdobramentos de pedalar em sentidos como visão e audição, bem como a possibilidade de compreender o uso da bicicleta como prática social ao se perceber o outro que pedala.

As categorias criadas vão ao encontro daquelas propostas por Heinen et al. (2010), ao discutirem os estudos a respeito do uso de bicicleta nos deslocamentos diários com base nas influências do ambiente construído, do ambiente natural, de variáveis socioeconômicas, dos fatores psicológicos e de aspectos como tempo, custo, segurança, etc. Dentre estes, os autores ressaltam a importância dos fatores psicológicos envolvidos no uso da bicicleta, em especial as atitudes, e sugerem pesquisas que aprofundem essa investigação.

De maneira geral, os artigos revisados exploram as variáveis relacionadas ao uso da bicicleta e apontam para a multideterminação do fenômeno. Incluem-se aspectos pessoais, ambientais e culturais e suas análises devem guardar as dimensões geográficas, históricas e sociais. Assim, em um contexto onde a geografia é favorável (por exemplo, o relevo é plano) e a cultura local favorece o uso da bicicleta como meio de transporte, é mais provável que esse modal seja visto de forma favorável. Isso pode ter desdobramentos positivos para as empresas locais, por exemplo, viabilizando bicicletários e vestiários aos funcionários, ou então nas políticas públicas, por meio da implementação de ciclovias. Por isso a advertência de Heinen et al. (2010) sobre a necessidade de cautela ao transferir os resultados dessas pesquisas para contextos diferentes.

As comparações de resultados referentes às variáveis sociodemográficas explicam a ressalva dos autores em relação à generalização. Por exemplo, os estudos convergem ao concluírem que ter um carro (Heinen et al., 2010), usar o carro (Zhang et al., 2014) e ter estacionamento grátis para carros no local de trabalho (Buehler, 2012) se relacionam negativamente com o uso de bicicleta. Por outro lado, acesso reduzido a carros (Hansen \& Nielsen, 2014), dificuldade de encontrar vagas para estacionar e a possibilidade de evitar congestionamentos (Franco et al., 2014) estimulam o uso da bicicleta. Portanto, as condições atreladas ao uso do carro (como congestionamentos e dificuldade de esta- 
cionar) parecem se relacionar positivamente com o uso da bicicleta, e não necessariamente ter acesso ao carro e às suas facilidades, aspectos que são característicos de segmentos populacionais, culturas e locais específicos.

Além disso, surgem divergências ao se comparar transporte com bicicletas e níveis educacionais. Heinen et al. (2010) identificam que pessoas com alta escolaridade pedalam menos para o trabalho, enquanto Hansen \& Nielsen (2014) relatam associação positiva entre esses fatores. Diante disso, aponta-se para a possibilidade de as particularidades culturais, econômicas e políticas dos locais de pesquisa influenciarem nos resultados. Análises produzidas em países europeus, onde o uso da bicicleta é bastante difundido, serão diferentes daquelas de países em desenvolvimento.

Em relação aos aspectos metodológicos, identificou-se a preponderância de estudos quantitativos que fazem uso de escalas e análises estatísticas para a apreensão do fenômeno, conforme a Tabela 2. Foram identificados 28 estudos nessa perspectiva, que contrastam com os cinco artigos que se orientam pela abordagem qualitativa. Daqueles, 17 trataram basicamente dos aspectos psicológicos envolvidos e é com base nisso que se discute a necessidade de aprofundar o entendimento sobre as características envolvidas com o fenômeno por meio de investigações de abordagem compreensiva (Heinen et al., 2010).

Parte dos estudos quantitativos foi feito com uso de questionários e escalas para acessar os fenômenos investigados, conforme destacado na Tabela 2. Neles os instrumentos foram variados e contemplaram escalas desenvolvidas especificamente para a pesquisa, escalas validadas em estudos anteriores e questionários utilizados em pesquisas de levantamento feitas anteriormente. Os estudos qualitativos privilegiaram a realização de grupos focais, entrevistas individuais semiestruturadas, a combinação entre as duas técnicas e o uso de gravações de áudio e vídeo.

Nos aspectos relacionados à psicologia como área de conhecimento e sua implicação na produção de estudos sobre a temática do uso da bicicleta, identificou-se que oito artigos relataram pesquisas conduzidas por psicólogos ou que incluíam na equipe membros oriundos desse campo do conhecimento, ainda que os descritores utilizados nas buscas tenham sido psicologia e psicologia ambiental. Assim, pesquisas sobre o uso de bicicletas podem consistir em terreno produtivo para pesquisadores da psicologia. Nesses termos, destaca-se a revisão realizada por Caballero et al. (2014) sobre a influência dos fatores psicológicos na escolha do uso da bicicleta como meio de transporte. Os autores apontam a intenção de pedalar como o principal determinante na escolha desse modal, associada com o apoio social percebido e a autoeficácia, corroborando os resultados desta revisão. Com relação aos modelos teóricos adotados nos estudos, não parece haver consenso nas pesquisas que abordam a temática do uso de bicicletas. Dos 33 artigos analisados, 18 não apresentaram fundamentação teórica explícita, enquanto cinco utilizaram-se da Teoria do Comportamento Planejado de Ajzen (1991) na sustentação dos resultados. Reflexo disso pode ser a falta de unidade ao se lidar com aspectos cognitivos e afetivos na escolha da bicicleta.

Nos estudos que tratam dessa temática, por vezes a cognição e os afetos aparecem como aspectos antagônicos, como negação um do outro (cognitivo é aquilo que não envolve afeto). Desse modo, a falta de integração teórica pode apontar para aspectos epistemológicos ao lidar com a esfera afetiva, o que evidencia a necessidade de aprofundamento a respeito do tema. 
TABELA2 - Identificação dos aspectos metodológicos das pesquisas sobre o uso da bicicleta como meio de transporte nos estudos pessoa-ambiente.

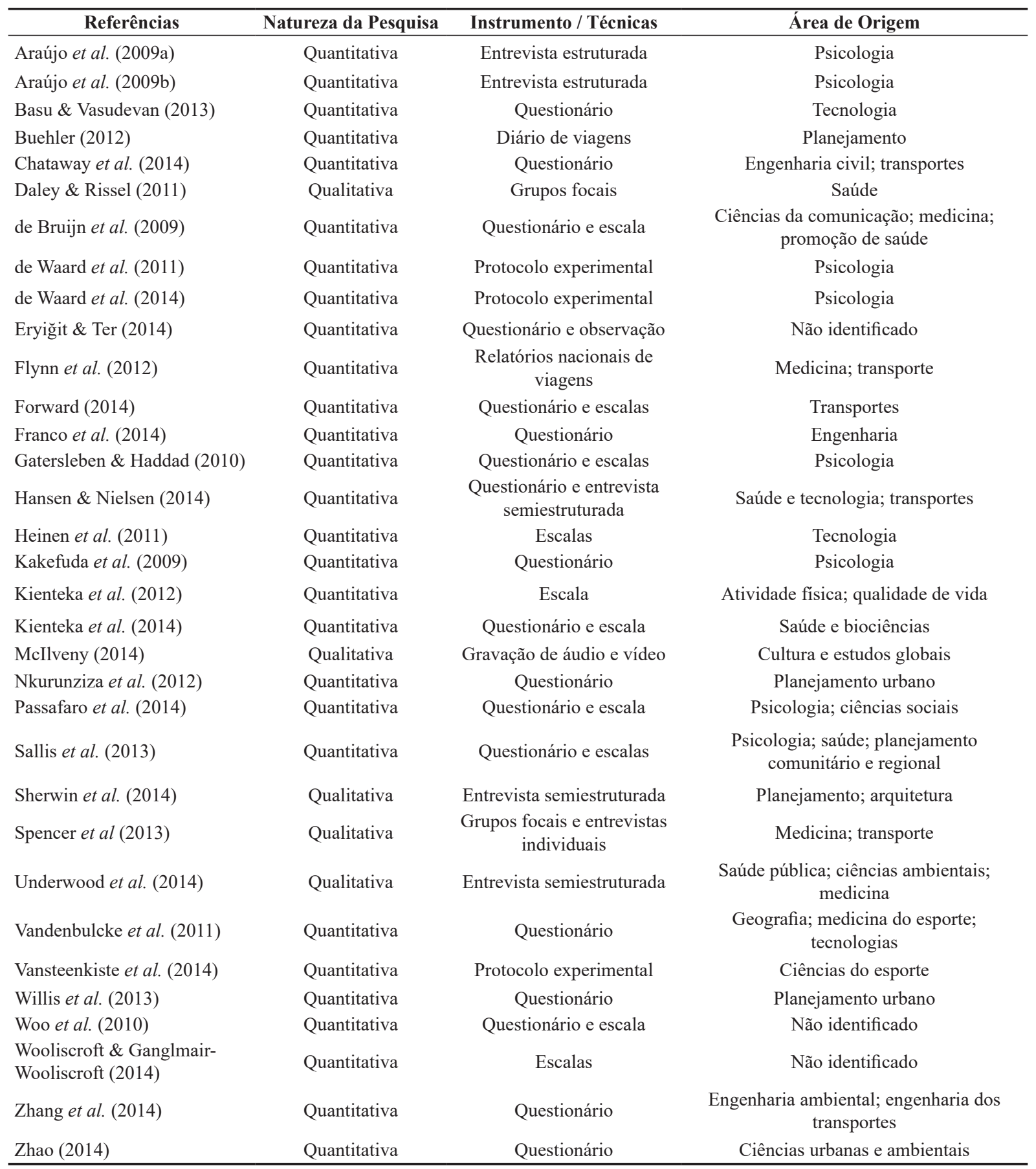

FONTE: Busca em bases de dados (2014). 
Destaca-se a relevância do ponto de vista dos ganhos psicológicos de se utilizar a bicicleta como meio de transporte. Os estudos destacam diminuição do estresse, experiências positivas e bom humor e a possibilidade de produção e fortalecimento da identidade, além da satisfação pessoal ao fazer a opção por esse modal.

Enfatiza-se a importância da compreensão da temática de maneira multideterminada e que percorre o campo interdisciplinar do conhecimento. Aprofundar o conhecimento de questões que dizem respeito à saúde e à qualidade de vida em contextos urbanos, como no caso da mobilidade urbana por bicicletas, pode auxiliar na elaboração de políticas públicas atentas às demandas não só de quem faz uso desse modal, mas de todos os participantes do trânsito.

A partir da identificação das variáveis associadas ao uso da bicicleta como meio de transporte (hábito, atitude, ambiente, comportamento e percepção), podem-se fazer algumas inferências. O hábito apresentou-se relacionado negativamente com a atitude, ou seja, quanto maior a intenção de usar a bicicleta, menor é o hábito de realmente

\section{Referências}

Ajzen, I. The theory of planned behavior. Organizational Behavior and Human Decision Processes, 50, 179-211, 1991. doi: 10.1016/0749-5978(91)90020-T

Amorim, L. C. de; Oliveira, G. M. de; Silva, A. N. R. da. Uma visão de mobilidade urbana sustentável segundo o discurso de pesquisadores e técnicos/gestores. In: XXVIII Congresso de Pesquisa e Ensino em Transportes da ANPET. Curitiba, Paraná, 24 a 28 de novembro de 2014. p. 1-12.

Araújo, M. R. M.; Sousa, D. A.; Oliveira, J. M.; Jesus, M. S.; Sá, N. R.; Santos, P. A. C.; Macedo Jr. R.; Lima, T. C. Andar de bicicleta: contribuições de um estudo psicológico sobre mobilidade. Temas em Psicologia, 17(2), 481-495, utilizá-la. Assim, a produção do hábito de uso desse modal, por meio de incentivos ambientais e políticos (como a diminuição das faixas de rolamento para veículos e a disposição de espaço na via para ciclistas e pedestres nos fins de semana, por exemplo), pode se apresentar como uma estratégia com desdobramentos positivos.

Além disso, o ambiente apresentado de forma segura para o ciclista é uma forma de manutenção do comportamento de uso da bicicleta para deslocamentos diários. Portanto, as políticas públicas que busquem uma perspectiva sustentável de mobilidade urbana precisam ter em vista os aspectos cognitivos e comportamentais das pessoas que fazem uso dos diversos modais de transporte, buscando a diversificação da oferta e da estrutura de acordo com a percepção dos usuários sobre os elementos ambientais. Por fim, indica-se a potencialidade socioambiental no uso de meios de locomoção "limpos", seja no nível político (do incentivo ao uso da bicicleta nas cidades) ou no aprimoramento da qualidade das relações que as pessoas estabelecem com seus entornos, fomentando condutas mais responsáveis. 2009a. Disponível em: <http://pepsic.bvsalud.org/pdf/tp/ v17n2/v17n2a18.pdf $>$.

Araújo, M. R. M.; Sousa, D. A.; Oliveira, J. M.; Jesus, M. S.; Sá, N. R.; Santos, P. A. C.; Macedo Jr. R.; Lima, T. C. Bicicleta e transferência modal: uma investigação em Aracaju. Temas em Psicologia, 17(2), 463-480, 2009 b. Disponível em: <http://pepsic.bvsalud.org/pdf/tp/v17n2/ v17n2a17.pdf $>$.

Basu, S.; Vasudevan, V. Effect of bicycle friendly roadway infrastructure on bicycling activities in urban India. Procedia - Social and Behavioral Sciences, 104, 1139-1148, 2013. doi: 10.1016/j.sbspro.2013.11.210 
Brasil. Lei n. 12.587, de 3 de janeiro de 2012. Institui as diretrizes da Política Nacional de Mobilidade Urbana. Brasília, DF: Presidência da República, Casa Civil, Subchefia para Assuntos Jurídicos. Disponível em: <http://www. planalto.gov.br/ccivil_03/_ato2011-2014/2012/lei/112587. $\mathrm{htm}>$. Acesso em: 29 set. 2014.

Buehler, R. Determinants of bicycle commuting in the Washington, DC region: the role of bicycle parking, cyclist showers, and free car parking at work. Transportation Research Part D, 17, 525-531, 2012. doi: 10.1016/j. trd.2012.06.003

Caballero, R.; Franco, P.; Mustaca, A.; Jakovcevic, A. Uso de la bicicleta como medio de transporte: influencia de los factores psicológicos. Una revisión de la literatura. Psico PUCRS, 45(3), 316-327, 2014. doi: 10.15448/19808623.2014.3.17286

Chataway, E. S.; Kaplan, S.; Nielsen, T. A. S.; Prato, C. G. Safety perceptions and reported behavior related to cycling in mixed traffic: a comparison between Brisbane and Copenhagen. Transportation Research Part F, 23, 32-43, 2014. doi: 10.1016/j.trf.2013.12.021

Daley, M.; Rissel, C. Perspectives and images of cycling as a barrier or facilitator of cycling. Transport Policy, 18, 211-216, 2011. doi: 10.1016/j.tranpol.2010.08.004

de Bruijn, G-J.; Kremers, S. P. J.; Singh, A.; van den Putte, B.; van Mechelen, W. Adult active transportation: adding habit strength to the Theory of Planned Behavior. American Journal of Preventive Medicine, 36(3), 189-194, 2009. doi: 10.1016/j.amepre.2008.10.019

de Waard, D.; Edlinger, K.; Brookhuis, K. Effects of listening to music, and of using a handheld and handsfree telephone on cycling behaviour. Transportation Research Part F, 14, 626-637, 2011. doi: 10.1016/j.trf.2011.07.001

de Waard, D.; Lewis-Evans, B.; Jelijs, B.; Tucha, O.; Brookhuis, K. The effects of operating a touch screen smartphone and other common activities performed while bicycling on cycling behaviour. Transportation Research Part F, 22, 196-206, 2014. doi: 10.1016/j.trf.2013.12.003

Doron, R.; Parot, F. (Orgs.). Comportamento. In: Dicionário de Psicologia. 1. ed. São Paulo: Ática, 2001. p. 157-158.

Eryiğit, S.; Ter, U. The effects of cultural values and habits on bicycle use - Konya sample. Procedia - Social and
Behavioral Sciences, 140, 178-185, 2014. doi: 10.1016/j. sbspro.2014.04.406

Flynn, B. S.; Dana, G. S.; Sears, J.; Aultman-Hall, L. Weather factor impacts of commuting to work by bicycle. Preventive Medicine, 54, 122-124, 2012. doi: 10.1016/j. ypmed.2011.11.002

Forward, S. E. Exploring people's willingness to bike using a combination of the theory of planned behavioral and the transtheoretical model. Revue Européenne de Psychologie Appliquée, 64, 151-159, 2014. doi: 10.1016/j. erap.2014.04.002

Franco, L. P. C.; Campos, V. B. G.; Monteiro, F. B. A characterization of commuter bicycle trips. Procedia - Social and Behavioral Sciences, 111, 1165-1174, 2014. doi: 10.1016/j. sbspro.2014.01.151

Gatersleben, B.; Haddad, H. Who is the typical bicyclist? Transportation Research Part F, 13, 41-48, 2010. doi: 10.1016/j.trf.2009.10.003

Günther, H. Mobilidade e affordance como cerne dos estudos pessoa-ambiente. Estudos de Psicologia, 8(2), 273-280, 2003. doi: 10.1590/S1413-294X2003000200009

Hansen, K. B.; Nielsen, T. A. S. Exploring characteristics and motives of long distance commuter cyclists. Transport Policy, 35, 57-63, 2014. doi: 10.1016/j.tranpol.2014.05.001

Heinen, E.; van Wee, B.; Maat, K. Commuting by bicycle: an overview of the literature. Transport Reviews, 30(1), 59-96, 2010. doi: 10.1080/01441640903187001

Heinen, E.; Maat, K.; van Wee, B. The role of attitudes toward characteristics of bicycle commuting on the choice to cycle to work over various distances. Transportation Research Part D, 16, 102-109, 2011. doi: 10.1016/j. $\operatorname{trd} .2010 .08 .010$

Kakefuda, I.; Stallones, L.; Gibbs, J. Discrepancy in bicycle helmet use among college students between two bicycle use purposes: commuting and recreation. Accident Analysis and Prevention, 41, 513-521, 2009. doi: 10.1016/j. aap.2009.01.014

Kienteka, M.; Rech, C. R.; Fermino, R. C.; Reis, R. S. Validade e fidedignidade de um instrumento para avaliar as barreiras para o uso de bicicleta em adultos. Revista Brasileira de Cineantropometria e Desempenho Humano, 14(6), 624-635, 2012. doi: 10.5007/1980-0037.2012v14n6p624 
Kienteka, M.; Reis, R. S.; Rech, C. R. Personal and behavioral factors associated with bicycling in adults from Curitiba, Paraná State, Brazil. Cad. Saúde Pública, 30(1), 79-87, 2014. doi: 10.1590/0102-311X00041613

McIlvenny, P. Vélomobile formations-in-action: biking and talking together. Space and Culture, 17(2), 137-156, 2014. doi: $10.1177 / 1206331213508494$

Nkurunziza, A.; Zuidgeest, M.; Brussel, M.; Van Maarseveen, M. Examining the potential for modal change: motivators and barriers for bicycle commuting in Dar-es-Salaam. Transport Policy, 24, 249-259, 2012. doi: 10.1016/j. tranpol.2012.09.002

Passafaro, P.; Rimano, A.; Piccini, M. P.; Metastasio, R.; Gambardella, V.; Gullace, G.; Lettieri, C. The bicycle and the city: desires and emotions versus attitudes, habits and norms. Journal of Environmental Psychology, 38, 76-83, 2014. doi: 10.1016/j.jenvp.2013.12.011

Rodrigues, A.; Assmar, E. M. L.; Jablonski, B. Psicologia Social. Petrópolis: Vozes, 1999.

Sallis, J. F.; Conway, T. L.; Dillon, L. I.; Frank, L. D.; Adams, M. A.; Cain, K.; Saelens, B. E. Environmental and demographic correlates of bicycling. Preventive Medicine, 57, 456-460, 2013. doi: 10.1016/j.ypmed.2013.06.014

Sherwin, H.; Chatterjee, K.; Jain, J. An exploration of the importance of social influence in the decision to start bicycling in England. Transportation Research Part A, 68, 32-45 2014. doi: 10.1016/j.tra.2014.05.001

Spencer, P.; Watts, R.; Vivanco, L.; Flynn, B. The effects of environmental factors on bicycle commuters in Vermont: influences of a northern climate. Journal of Transport Geography, 31, 11-17, 2013. doi: 10.1016/j.jtrangeo.2013.05.003

Underwood, S. K.; Handy, S. L.; Paterniti, D. A.; Lee, A. E. Why do teens abandon bicycling? A retrospective look at attitudes and behaviors. Journal of Transport \& Health, 1, 17-24, 2014. doi: 10.1016/j.jth.2013.12.002

Vandenbulcke, G; Dujardin, C.; Thomas, I.; de Geus, B.; Degraeuwe, B.; Meeusen, R.; Panis, L. I. Cycle commuting in Belgium: spatial determinants and 're-cycling' strategies. Transportation Research Part A, 45, 118-137, 2011. doi: 10.1016/j.tra.2010.11.004

Vansteenkiste, P.; Zeuwts, L.; Cardon, G.; Philippaerts, R.; Lenoir, M. The implications of low quality bicycle paths on gaze behavior of cyclists: a field test. Transportation Research Part F, 23, 81-87, 2014. doi: 10.1016/j. trf.2013.12.019

Vasconcellos, E. A. Mobilidade urbana e cidadania. Rio de Janeiro: Senac Nacional, 2012.

Villada, C. A. G.; Portugal, L. da S. Mobilidade sustentável e o desenvolvimento orientado ao transporte sustentável. In: Anais do XXIX Congresso de Pesquisa e Ensino em Transportes da ANPET, Ouro Preto, Minas Gerais, 09 a 13 de novembro de 2015. p. 2743-2754.

Willis, D. P.; Manaugh, K.; El-Geneidy, A. Uniquely satified: exploring cyclist satisfaction. Transportation Research Part F, 18, 136-147, 2013. doi: 10.1016/j.trf.2012.12.004

Woo, T. C.; Helton, W. S.; Russel, P. N. The role emotion and gender play in the choice of commuting by bicycle: a preliminary study from Christchurch, New Zealand. Proceedings of the Human Factors and Ergonomics Society Annual Meeting, 54, 1067-1071, 2010. doi: 10.1177/154193121005401404

Wooliscroft, B.; Ganglmair-Wooliscroft, A. Improving conditions for potential New Zealand cyclists: an application of conjoint analysis. Transportation Research Part A, 69, 11-19, 2014. doi: 10.1016/j.tra.2014.08.005

Zhang, D.; Magalhães, J. D. A. V.; Wang, X. Prioritizing bicycle paths in Belo Horizonte City, Brazil: analysis based on user preferences and willingness considering individual heterogeneity. Transportation Research Part A, 67, 268278, 2014. doi: 10.1016/j.tra.2014.07.010

Zhao, P. The impact of the built environment on bicycle commuting: evidence from Beijing. Urban Studies, 51(5), 1019-1037, 2014. doi: 10.1177/0042098013494423 